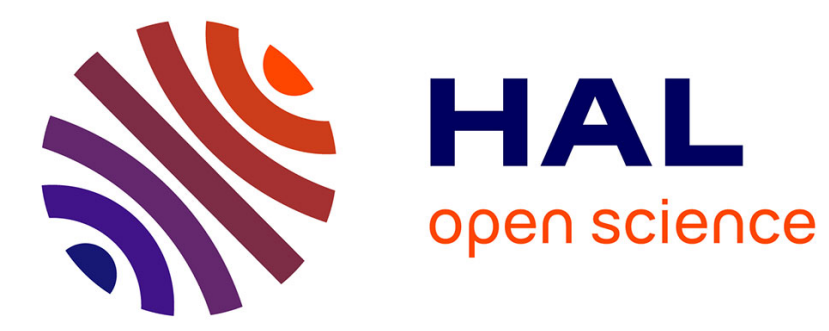

\title{
Dusty plasmas: synthesis, structure and dynamics of a dust cloud in a plasma
}

Maxime Mikikian, Lénaïc Couëdel, Marjorie Cavarroc, Yves Tessier, Laifa Boufendi

\section{- To cite this version:}

Maxime Mikikian, Lénaïc Couëdel, Marjorie Cavarroc, Yves Tessier, Laifa Boufendi. Dusty plasmas: synthesis, structure and dynamics of a dust cloud in a plasma. European Physical Journal: Applied Physics, 2010, 49, pp.13106. 10.1051/epjap/2009191 . hal-00436503

\section{HAL Id: hal-00436503 https://hal.science/hal-00436503}

Submitted on 26 Nov 2009

HAL is a multi-disciplinary open access archive for the deposit and dissemination of scientific research documents, whether they are published or not. The documents may come from teaching and research institutions in France or abroad, or from public or private research centers.
L'archive ouverte pluridisciplinaire HAL, est destinée au dépôt et à la diffusion de documents scientifiques de niveau recherche, publiés ou non, émanant des établissements d'enseignement et de recherche français ou étrangers, des laboratoires publics ou privés. 


\title{
Dusty plasmas: synthesis, structure and dynamics of a dust cloud in a plasma
}

\author{
M. Mikikian ${ }^{\mathrm{a}}$, L. Couëdel ${ }^{\mathrm{b}}$, M. Cavarroc ${ }^{\mathrm{c}}$, Y. Tessier, and L. Boufendi \\ GREMI, Groupe de Recherches sur l'Énergétique des Milieux Ionisés, UMR 6606, CNRS/Université d'Orléans, \\ 14 rue d'Issoudun, BP 6744, 45067 Orléans Cedex 2, France
}

Received: 21 June 2009 / Accepted: 9 October 2009

Published online: 26 November 2009 - (c) EDP Sciences

\begin{abstract}
Plasmas are energetic media that can give birth to dust particles due to the presence of reactive gases or plasma-surface interactions. Industrial plasmas are often concerned by these dust particles that can be either unwanted or useful for the process. For fusion plasmas, production of dust particles from wall erosion is a serious issue for performance and safety reasons. In this article, some aspects of dusty plasmas with potential implications for plasma experimenters will be discussed. Convenient ways for detecting the presence or the growth of dust particles will be presented. The spatial distribution of the dust cloud during the plasma phase determines the subsequent dust particle deposition. It will be shown that some reactor regions can attract or repeal these dust particles. Finally, the dust particle dynamics after the plasma extinction will be investigated. A special attention will be paid on the residual electric charge that can stay attached on the dust particle surface and on its implications for dust particle control or deposition.
\end{abstract}

PACS. 52.27.Lw Dusty or complex plasmas; plasma crystals

\section{Introduction}

Plasmas are found in many natural environments, particularly in astrophysics, and are also used for their versatile properties in various industrial processes. They often coexist with dust particles (solid bodies with sizes ranging from a few $\mathrm{nm}$ to $\mathrm{cm}$ ) and these new media are then called dusty (or complex) plasmas [1]. Dust particles can be formed from reactive gases [2] (like silane [3-9], methane [10-13] or acetylene $[10,14-16]$ ) or material sputtering [17-22] (providing molecular precursors or already formed solid bodies) through a complex succession of chemical and physical reactions. Dust particles usually attach plasma free electrons inducing a new plasma equilibrium and the occurrence of new phenomena.

Comet tails or planetary atmospheres are natural dusty plasmas (for example, methane in Titan's atmosphere is responsible of aerosol formation [23]). In industry, dust particles are often considered as fatal for the processes, in particular in microelectronics where extreme cleanliness is required. Thus, important investments are engaged in order to work under a controlled and filtered environment, free of dust particle contamination. Despite of these efforts, it appears that dust particles can arise from the plasma process itself. Indeed, in the 1980s, dust particle

\footnotetext{
a e-mail: maxime.mikikian@univ-orleans.fr

b Present address: Max-Planck-Institut für Extraterrestrische Physik, Giessenbachstrasse, 85741 Garching, Germany.

Present address: Made In Dreux, 4 rue Albert-Caquot, 28500 Vernouillet, France.
}

formation in the gas phase has been evidenced in reactors used for silicon based device fabrication [24]. Thus, many studies $[4-7,25]$ began for understanding dust particle growth and behavior in order to avoid either their formation or deposition.

Another field where dust particles are a serious issue, is thermonuclear fusion. Indeed, in fusion devices like ITER, reactor walls can be severely eroded by the plasma. The physical and chemical erosions of plasma facing materials can produced dust particles [26-28] which strongly reduce performances of the fusion plasma. Moreover, dust particles can have a porous structure which facilitate the trapping of the tritium. Thus, production of dust particles has to be minimized and the secure and easy removal of this radioactive material has to be optimized.

In opposition to these negative aspects, dusty plasmas also appear to be potentially useful, for example in nanotechnology. Indeed, these media are very competitive tools for depositing nanostructured films or for building nanometer size devices in a "bottom-up" approach. In this direction, controlled formation of silicon nanocrystals [29] became of high interest because they can be used for enhancing optoelectronic properties of deposited films in solar cell technology [30] and for building single-electron devices (SED) like transistors or memories [31]. Carbon based materials are also investigated by using hydrocarbon gases $[2,12]$. They are involved in the deposition of diamond-like carbon (DLC) films [32] or nanocrystalline diamond used in industry for their unique properties like extreme hardness. 


\section{Experimental setups}

In this work, dusty plasmas are created in two capacitively coupled radiofrequency discharges (cc-rf) with different geometries and experimental conditions. One reactor [6] is dedicated to the study of dust particle formation in silane-based chemistries with a particular attention paid to the controlled growth of single-crystal silicon nanoparticles $\left(r_{d} \sim 2 \mathrm{~nm}\right)$ [29]. Its design is close to some typical industrial reactors. The working pressure is about $10 \mathrm{~Pa}$ and the power around $10 \mathrm{~W}$. The second reactor is the PKE-Nefedov device [33] where dust particles are formed by sputtering a polymer layer exposed to an argon plasma [21]. The pressure is around $150 \mathrm{~Pa}$ and the typical power is $3 \mathrm{~W}$. It is equipped with glass walls convenient for an easy visualization of the dust cloud. Main studies concern dust particle formation, dust cloud dynamics and various unstable phenomena induced by the presence of dust particles.

Many results are qualitatively similar in both reactors, meaning that after the first instants of dust particle formation, where chemistry is the prevailing process, the physics is quite the same. Thus, described phenomena are certainly frequent in many typical low pressure plasmas.

\section{Dust detection}

In laboratory or industrial reactors, the presence of dust particles formed from reactive gases or sputtering can be revealed by several ways, either directly or indirectly. The most obvious method is the direct visualization of the dust particles by using laser light scattering (LLS). In the late 1980s, this method revealed the presence in industrial plasma reactors of huge dust particle clouds trapped close to the sheath boundaries above wafers [24]. This discovery was at the origin of a strong growing interest for dusty plasmas outside the astrophysics community. However, LLS is not a very convenient method in industry because it requires at least two optical accesses, one for the laser (as a beam or a sheet) and one for recording the scattered light (often at $90^{\circ}$ from laser direction). Thus, for this type of application, a more suitable diagnostics has to evidence the presence of dust particles thanks to only one small optical access or even better, without any. This detection can be performed in an indirect way, through the plasma modifications induced by dust particles [34]. Indeed, in typical laboratory or industrial plasmas, dust particles tend to acquire a negative charge $Q_{d}$ proportional to their radius $r_{d}$. Assuming the simple model of a spherical capacitor, it gives

$$
Q_{d}=4 \pi \epsilon_{0} r_{d} V_{d}
$$

where $V_{d}$ is the dust surface potential (like the floating potential of a spherical Langmuir probe) relatively to the plasma (or more generally the local) potential. Thus, when the couple of number density and $r_{d}$ affects significantly the free electron density $n_{e}$, plasma characteristics change. This effect can be observed on both optical and electrical

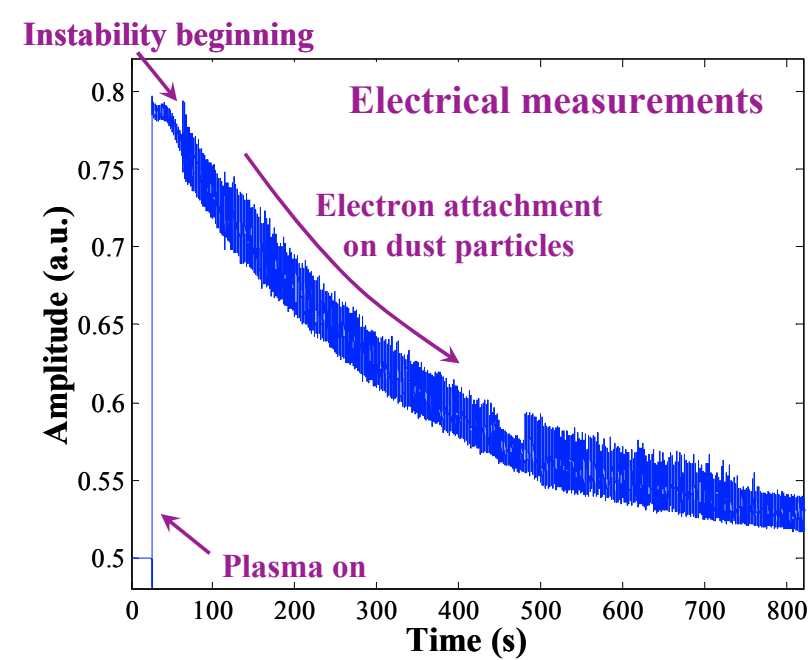

Fig. 1. (Color online) Decrease of the discharge current during dust particle growth in the PKE-Nefedov reactor. When the dust particle effect strongly changes the plasma equilibrium, instabilities can occur.

properties of the discharge and allows to detect and follow the dust particle growth process. The decrease of the electron density is often related with an increase of the electron temperature $T_{e}$ [35]. These changes appear as a decrease of the discharge current which is easily measurable (Fig. 1) and as an enhancement of the plasma light emission which is detectable with only one optical access. These two plasma modifications have to attract the experimenter attention on the possible presence of growing dust particles in the discharge. When dust particle formation is the process objective, these diagnostics provide useful information on dust particle growth kinetics.

If the grown dust density is relatively high, the electron depletion is important and the plasma equilibrium is severely disturbed. In these conditions, low frequency instabilities $[20,29,36-39]$ (from a few $\mathrm{Hz}$ to a few $\mathrm{kHz}$ ) can be observed in all types of measurement and be used as a diagnostics. Indeed, this global unstable phenomenon appears clearly on both electrical [36-38] (Fig. 1) and optical $[20,38,39]$ (Fig. 2) signals. On the optical signal, these instabilities correspond to spatial and temporal fluctuations of the plasma light. Some regions with enhanced light emission appear successively in different parts of the plasma as observed in Figure 2.

The shape and frequency of these unstable phases are strongly related to dust particle size and number density $n_{d}$. In particular, the instability frequency is usually related to the dust plasma frequency $\omega_{p d}[36]$, defined similarly to the classical electron and ion plasma frequencies as

$$
\omega_{p d}=\sqrt{\frac{n_{d} Q_{d}^{2}}{\epsilon_{0} m_{d}}},
$$

with $m_{d}$ the dust particle mass. Thus, when dust particles are growing in the plasma, these instabilities evolve [38] and characterize the growth kinetics. In silane based plasmas, they have been shown to be a powerful 


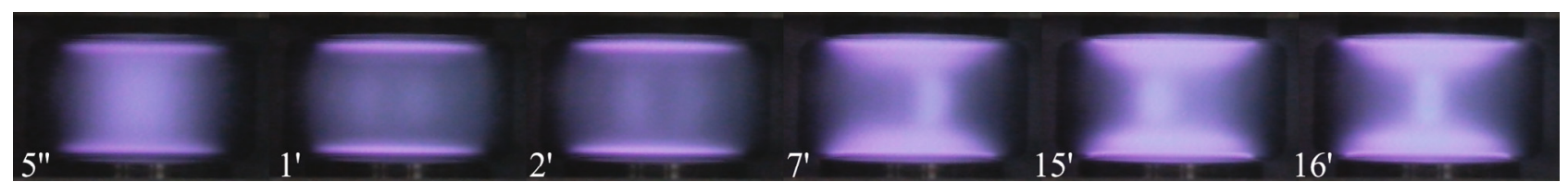

Fig. 2. (Color online) Argon plasma light modifications during instabilities induced by dust particle growth in the PKE-Nefedov reactor. From left to right, frames taken at different instants from plasma ignition to 16 min. The distance between the two electrodes is about $3 \mathrm{~cm}$ and the two bright regions observed on top and bottom of each image correspond to the presheath regions.

tool to indicate the first coagulation step where individual crystalline nanoparticles of about $2 \mathrm{~nm}$ start to form aggregates [29]. This sudden increase in size creates a drastic change in the global electric charge carried by the dust cloud (as $Q_{d} \propto r_{d}$ ) which perturbs the plasma equilibrium. A possible explanation for this phenomenon is the attachment induced ionization instabilities observed in low pressure rf discharges in electronegative gases [40,41]. As dust particles are charged an analogy can be suggested, considering that they behave like large negative ions. Localization of this coagulation step can be useful for potential applications requiring non agglomerated nanocrystals of about $2 \mathrm{~nm}$ in radius (for example SED or polymorphous silicon for solar cells). Indeed, this moment indicates when the experimenter has to switch off the discharge to deposit these nanocrystals on a substrate. The density of deposited nanocrystals can thus be controlled by repeating the experiment several times, for example by pulsing the discharge.

\section{Dust cloud structure}

If dust particles are detected by one of the above diagnostics and are potentially harmful for the process, it is necessary to know their spatial distribution in the discharge. Indeed, position and structure of the dust cloud are controlled by several forces like gravity, neutral drag, thermophoresis (pushing dust particles away from hot regions) and, as dust particles are usually electrically charged, electric forces and ion drag. In the reactor, the equilibrium between these forces is different from one place to another and thus, some regions can attract, repeal or trap the dust particle cloud.

Figure 3 shows a dust cloud $\left(r_{d} \sim\right.$ a few hundreds of $\mathrm{nm}$ ) observed thanks to LLS in the PKE-Nefedov reactor. The dust cloud is trapped in the discharge due to the strong electric field existing in the sheathes. As the plasma potential is usually higher than the wall or electrode potential, the electric field is directed towards the surrounding walls. Thus, the negatively charged dust particles are confined in the plasma. As an example, Figure 3 clearly shows that dust particles cannot enter deeply in the sheath and levitate at a minimum distance of around $2.5 \mathrm{~mm}$ above the lower electrode. This effect explains that dust particles can accumulate above wafers during the plasma phase in industrial processes [24]. Then, when the plasma is switched off, the trapping electric forces dis-

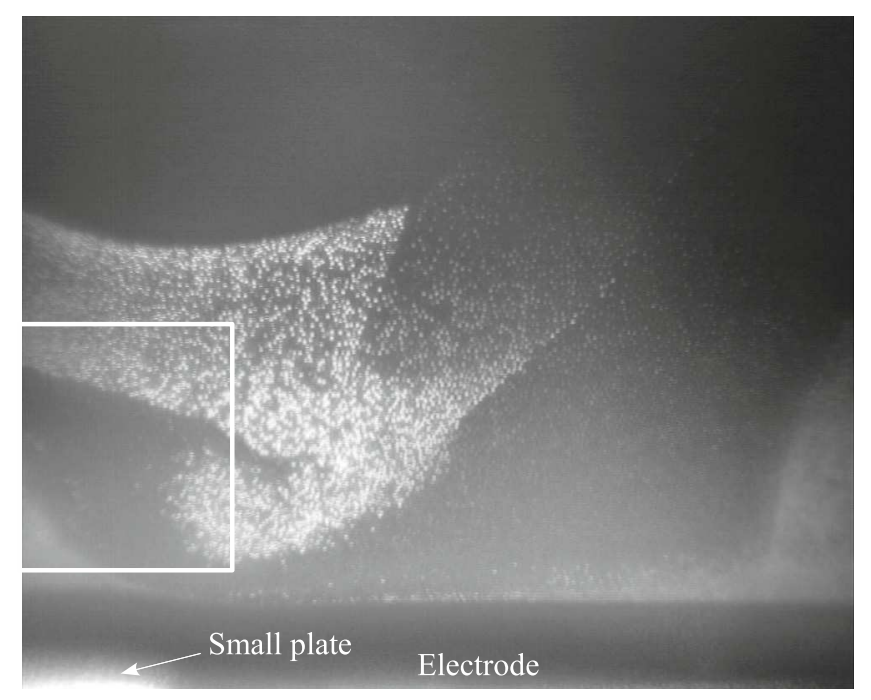

Fig. 3. Trapping of a dust cloud by the sheath electric field in the PKE-Nefedov reactor. Electrostatic perturbations of the cloud (induced by the electrode edge and by a thin plate placed on the lower electrode) are visible. The white rectangle corresponds to the field of view shown in Figure 4.

appear and dust particles can fall down and contaminate the wafers.

As charged dust particles are very sensitive to electric fields, any spatial inhomogeneity of this field can strongly influence the dust particle confinement. These inhomogeneities are observed close to immersed surfaces with steps or corners close to the plasma region. In typical ccrf discharges, it is well known that electric field lines are strongly curved near the edge of the electrodes. This effect can be seen in Figure 3 in the lower right part of the image, just above the sheath where dust particle trajectories follow vortex-like motions (see also [21] for a better description of this effect). These vortices are observed close to electric field steps. A small metallic plate (that could represent a substrate in a process) has been placed close to the center of the lower electrode (Fig. 3). It induces a small potential step that strongly influences the dust particle cloud uniformity and reveals its high sensitivity to any electric field. A zoom of the dust cloud region above the plate has been performed using a camera with a higher magnification lens (Fig. 4). Some dust particles are trapped above this plate (left part of the image) and the plate edge induces vortex motions (right part) like on the electrode edge. These observations clearly illustrate 


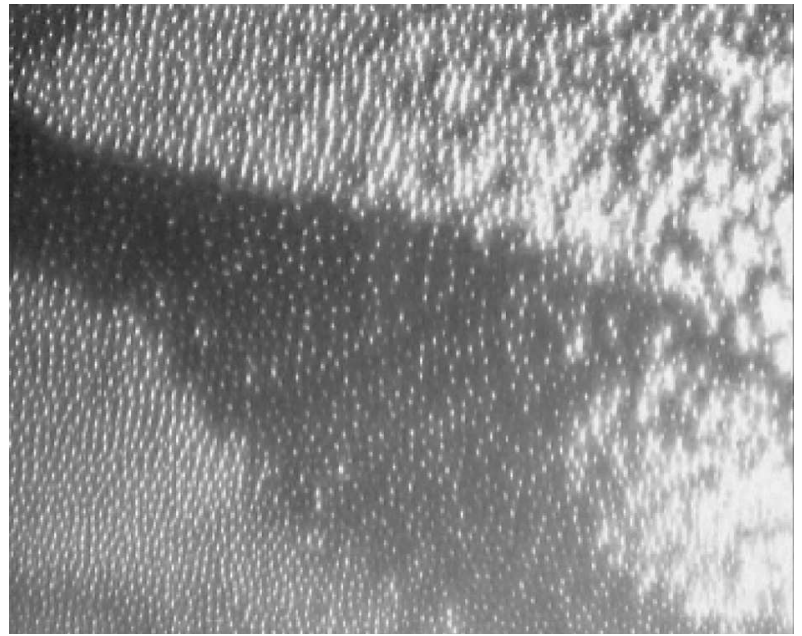

Fig. 4. Dust cloud perturbation induced by a thin plate placed on the lower electrode (zoom of the region indicated in Fig. 3).

that some reactor regions are traps for dust particles. This sensitivity to electric fields has to be considered by the experimenters dealing with dust particles trapped inside a plasma, in order to control or avoid their deposition. In addition, this property can be useful to develop electrostatic dust removal techniques [42-44] in industrial or fusion plasma devices.

It has been shown that, due to their charge, dust particles are highly sensitive to electric fields. This negative charge also attracts the plasma positive charges i.e. the positive ions. Thus, positive ions exert an additional force on dust particles by directly impacting the dust particle surface or by Coulomb collisions. This ion drag force is very important close to the sheathes because of the local electric field which accelerates the ions towards the electrodes (thus opposed to dust particle trapping).

The ion drag force is also involved in another typical dusty plasma phenomenon. Indeed, due to the geometry of typical cc-rf discharges, a dust-free region is often observed close to the discharge center (Fig. 5). This region, called the "void" $[9,20,21,45-50]$ is suspected to be sustained by two counteracting forces, an inward electric force and an outward ion drag force [46]. The ion drag force expels the dust particles from the plasma center while the electric force acts as a restoring force. The place where these two forces equilibrate, defines the void boundary.

The presence of the void region can be of high importance for experimenters concerned with trapped dust cloud. As an example, the void can mislead the experimenter trying to detect the presence of harmful dust particles by LLS. Indeed, LLS measurements are often performed in the plasma center and the lack of detected light could be interpreted as the absence of dust particles, whereas they surround the analyzed region. Thus, wrong conclusions about the presence of dust particles can be deduced. Another situation is when dust particles are required for deposition on a substrate. Each part of

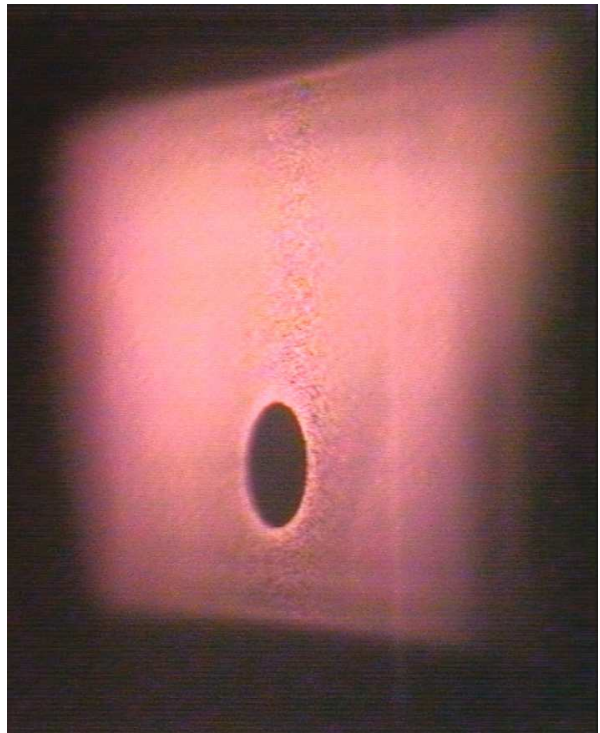

Fig. 5. (Color online) Dust-free region (void) in the center of a dust cloud in the PKE-Nefedov reactor. Due to the small angle between the laser direction and the camera position, a perspective effect is observed. The surrounding dark parts mark the boundaries of the dust cloud, the upper one corresponding to the sheath of the upper electrode (the lower one corresponds in fact to the limit of the laser sheet).

this substrate collects the dust particles contained in the perpendicular volume element. As the void corresponds to a strong inhomogeneity in the dust cloud structure, the substrate zone directly below the void will collect less dust particles. Thus, the inhomogeneity in the dust cloud structure is transfered to the deposited layer.

\section{Behavior of dust particles once the plasma is switched off}

Whether dust particles are useful or harmful, their behavior after the plasma extinction is of great importance in order to know where they are transported. Indeed, when the plasma is turned off, dust particles will be no longer trapped in the discharge by the confining electric fields and their behavior will depend on the remaining forces like [1]:

- Gravity inducing their wanted or unwanted deposition on the lower substrate or electrode

$$
F_{g}=\frac{4}{3} \pi r_{d}^{3} \rho g
$$

with $\rho$ the dust particle mass density.

- Gas flow (neutral drag), even small flows can transport dust particles and bring them in various reactor regions or in the pumping system with damage risks

$$
F_{n d}=-\frac{4}{3} \pi r_{d}^{2} m_{n} n_{n} v_{t h}\left(1+\alpha \frac{\pi}{8}\right)\left(\mathbf{v}_{\mathbf{d}}-\mathbf{v}_{\mathbf{n}}\right),
$$




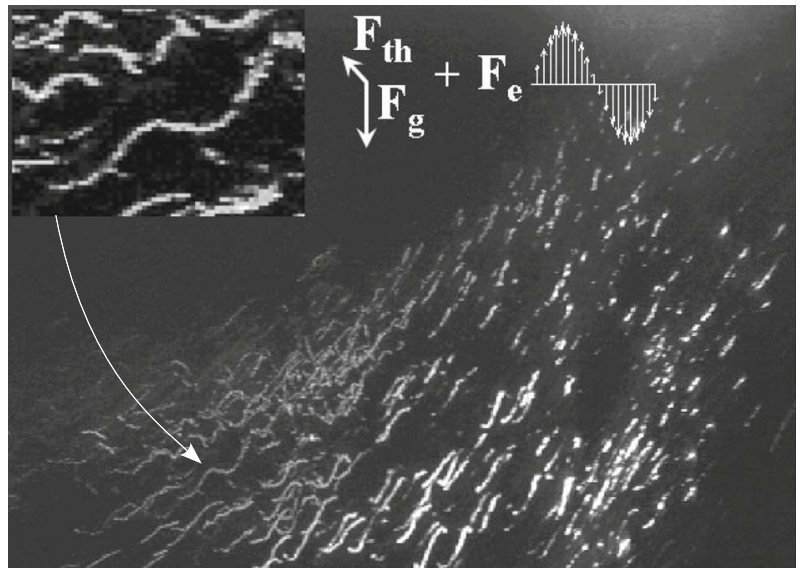

Fig. 6. Dust particle dynamics after the plasma shutdown under the action of different forces: gravity, thermophoresis, oscillating electric force. The neutral drag force damps the dust particle motion. The upper left insert is a zoom of the region marked by an arrow.

with $m_{n}, n_{n}$ and $v_{t h}=\sqrt{8 k_{B} T_{n} / \pi m_{n}}$ the mass, density and thermal speed of neutrals $\left(T_{n}\right.$ being the gas temperature), $v_{d}$ the dust particle velocity, $v_{n}$ the neutral mean speed and $\alpha$ the accommodation coefficient which is zero for specular reflection and unity for perfect diffuse reflection (this last case appears to be valid in typical laboratory experiments).

- Thermophoresis pushing dust particles away from hot surfaces like the powered electrode

$$
F_{T}=-\frac{32}{15} r_{d}^{2} \frac{k_{t r}}{v_{t h}} \nabla T
$$

where $k_{t r}$ is the translational part of the thermal conductivity and $\nabla T$ the temperature gradient.

To illustrate some of these forces, Figure $6\left(r_{d} \sim\right.$ a few hundreds of $\mathrm{nm}$ ) shows the dynamics of dust particles after the plasma extinction in the central part of the PKE-Nefedov reactor. This figure is obtained by superimposing several images and making visible dust particles trajectories. In this case, gravity and thermophoresis are counteracting. As these experiments are performed with a static pressure i.e. no gas flow, $v_{n}=0$ in equation (4) and thus the neutral drag acts as a friction force damping the dust particle motion. Thermophoresis was voluntarily created by cooling down the upper electrode for inducing a force opposed to gravity. Here, the equilibrium between these two forces is not reached and gravity is the dominant force inducing the fall of the dust particles. It is well observed that a small thermal gradient in the horizontal direction also exists, explaining the drift towards the left hand side of the image (Fig. 6). As dust particles are not perfectly monodisperse in the case presented in Figure 6, slight differences between their trajectories can be observed. Small thermal gradients can have important effects on dust particle dynamics. Indeed, in the afterglow, equalizing equations (3) and (5) indicates that a perfect compensation of gravity by thermophoresis could be ob- served for a dust particle of radius:

$$
r_{d}=\frac{8}{5 \pi \rho g} \frac{k_{t r}}{v_{t h}} \nabla T .
$$

As an example, if we consider a carbon graphite dust particle $\left(\rho_{d} \simeq 2260 \mathrm{~kg} \mathrm{~m}^{-3}\right)$ in argon in the PKE-Nefedov reactor with a interelectrode distance of $\simeq 3 \mathrm{~cm}$, a thermal gradient of only $1 \mathrm{~K} / \mathrm{cm}$ (a difference of 3 degrees between the two electrodes) is sufficient to counterbalance gravity for a dust particle of $r_{d} \simeq 100 \mathrm{~nm}$. This numerical application gives close results if we considered a silicon dust particles in argon because the mass density is similar $\rho_{d} \simeq 2330 \mathrm{~kg} \mathrm{~m}^{-3}$.

Figure 6 also reveals that electric forces can still have an action on dust particles after the plasma extinction. Indeed, until recently, it was supposed that dust particles loose their electric charge as soon as the plasma ends. However, it has been shown recently that dust particles can keep a residual charge of a few elementary charges (instead of several hundreds for a $100 \mathrm{~nm}$ dust particle during the plasma phase) $[51,52]$. This residual charge has been evidenced by observing that, once the plasma is off, dust particles can still follow a low frequency $(\sim 1 \mathrm{~Hz})$ oscillating (sinusoidal) electric field (Fig. 6 and zoom in the insert) specially applied for this investigation. The oscillating potential was applied on the lower electrode with an amplitude of about $\pm 30 \mathrm{~V}$. This oscillating response is superimposed on the trajectory defined by gravity and thermophoresis. In order to quantify this residual charge, oscillating amplitudes have been measured during experiments where gravity is perfectly balanced by thermophoresis. Thus, the equation of motion of dust particles contains only the applied electric force and the neutral drag. Resolving this equation leads to the following expression for the oscillation amplitude $A$ :

$$
A=\frac{Q_{d} E_{0}\left(z_{\text {mean }}\right)}{m_{d} \omega \sqrt{\omega^{2}+\frac{\beta^{2}}{m_{d}^{2}}}},
$$

where $E_{0}\left(z_{\text {mean }}\right)$ is the electric field value at the mean levitation height, $\omega=2 \pi f$ the electric field pulsation, and $\beta$ is a damping coefficient containing the multiplicative terms of equation (4). In our experimental conditions, oscillations with amplitudes around $1 \mathrm{~mm}$ can be obtained.

More surprising, the oscillation phase shows that this charge can be either negative or positive [52-56]. By analyzing statistically the value of this charge on many dust particles, the residual charge distribution appears to be highly dependent on the complex diffusion processes of electrons and ions during the afterglow period [53,55]. Indeed, in typical laboratory plasmas, the dust particle charge during the plasma phase is always negative. Nevertheless, during the afterglow the diffusion processes of ions and electrons can lead to a situation where, during a short period of time, dust particles are immersed in a medium mainly constituted of positive ions fixing their charge (electrons are lighter and can leave the medium faster than ions during the free diffusion regime [53]). Thus, in the afterglow, electrostatic interactions between 
dust particles can occur and agglomeration could be possible between dust particles with opposite charges.

Several interests arise for the study of this residual charge:

- This residual charge can be harmful in industrial applications dealing with nanoparticle deposition for nanostructured materials or single-electron devices. Indeed, a residual charge on deposited nanocrystals could be the cause of dysfunctions in the designed systems.

- This residual charge can strongly modify the dust particle dynamics during the afterglow. Any electric field can transport them towards unexpected regions and thus, for example, can avoid their deposition on the desired substrate.

- This residual charge could be useful for developing an easy electrostatic method for dust particle removal once the plasma has been switched off. In this case, as the plasma is off, the process is not disturbed by the removal technique as it could happen with other methods working during the plasma phase [42-44]. Thus, the use of this possible residual charge for developing new dust particle removal methods is of interest for both industry and fusion devices like ITER. If they are still charged, techniques based on the residual charge could open new perspectives for cleaning.

\section{Conclusion}

In this paper, several aspects of dusty plasma physics related to industrial or fusion plasmas are addressed. Different simple ways to detect wanted (or unwanted) dust particles are reported taking into account their quite easy implementation on many plasma devices. If a high density of dust particles is detected, the dust cloud location and structure are highly dependent of the various forces in presence. It has been shown that due to their high electric charge during the plasma phase, dust particles can easily be attracted or repealed by any local electrical field. This effect can produce traps for dust particles in different regions of the reactor. Some plasma forces are responsible of the formation of a dust-free region close to the plasma center. This void has the drawbacks to potentially mislead the experimenter using LLS in the plasma center to detect dust particles or to create inhomogeneous depositions. Finally, it has been shown that dust particle dynamics once the plasma has been switched off, has to be finely characterized to allow or avoid dust particle deposition. The attention has been especially drawn to recent results showing that dust particles can still be charged. It can have impact on their dynamics or on the performance of designed devices like SED. This property could also open new perspectives for the design of future dust particle removal methods.

The authors would like to thank S. Dozias and B. Dumax for electronic support and J. Mathias for optical support. The PKE-Nefedov chamber has been made available by the Max-Planck-Institute for Extraterrestrial Physics, Germany, under the funding of DLR/BMBF under grants No. 50WM9852. This work was supported by CNES under contract $02 / \mathrm{CNES} / 4800000059$.

\section{References}

1. P.K. Shukla, A.A. Mamun, Introduction to Dusty Plasma Physics (IOP Publishing, Bristol, 2002)

2. J. Berndt, E. Kovačević, I. Stefanović, O. Stepanovic, S.H. Hong, L. Boufendi, J. Winter, Contrib. Plasma Phys. 49, 107 (2009)

3. R.M. Roth, K.G. Spears, G.D. Stein, G. Wong, Appl. Phys. Lett. 46, 253 (1985)

4. Y. Watanabe, M. Shiratani, Y. Kubo, I. Ogawa, S. Ogi, Appl. Phys. Lett. 53, 1263 (1988)

5. A.A. Howling, C. Hollenstein, P.J. Paris, Appl. Phys. Lett. 59, 1409 (1991)

6. A. Bouchoule, A. Plain, L. Boufendi, J.P. Blondeau, C. Laure, J. Appl. Phys. 70, 1991 (1991)

7. P. Belenguer, J.P. Blondeau, L. Boufendi, M. Toogood, A. Plain, A. Bouchoule, C. Laure, J.P. Boeuf, Phys. Rev. A 46, 7923 (1992)

8. U.V. Bhandarkar, M.T. Swihart, S.L. Girshick, U.R. Kortshagen, J. Phys. D: Appl. Phys. 33, 2731 (2000)

9. M. Cavarroc, M. Mikikian, Y. Tessier, L. Boufendi, Phys. Rev. Lett. 100, 045001 (2008)

10. C. Deschenaux, A. Affolter, D. Magni, C. Hollenstein, P. Fayet, J. Phys. D: Appl. Phys. 32, 1876 (1999)

11. S. Hong, J. Berndt, J. Winter, Plasma Sources Sci. Technol. 12, 46 (2003)

12. J. Pereira, I. Géraud-Grenier, V. Massereau-Guilbaud, A. Plain, V. Fernandez, Surf. Coat. Technol. 200, 6414 (2006)

13. V. Massereau-Guilbaud, J. Pereira, I. Géraud-Grenier, A. Plain, J. Appl. Phys. 105, 033302 (2009)

14. I. Stefanović, E. Kovačević, J. Berndt, J. Winter, New J. Phys. 5, 39 (2003)

15. K. De Bleecker, A. Bogaerts, W. Goedheer, Phys. Rev. E 73, 026405 (2006)

16. E. Kovačević, J. Berndt, I. Stefanović, H.W. Becker, C. Godde, T. Strunskus, J. Winter, L. Boufendi, J. Appl. Phys. 105, 104910 (2009)

17. G.M. Jellum, D.B. Graves, J. Appl. Phys. 67, 6490 (1990)

18. G.S. Selwyn, J.S. McKillop, K.L. Haller, J.J. Wu, J. Vac. Sci. Technol. A 8, 1726 (1990)

19. B. Ganguly, A. Garscadden, J. Williams, P. Haaland, J. Vac. Sci. Technol. A 11, 1119 (1993)

20. G. Praburam, J. Goree, Phys. Plasmas 3, 1212 (1996)

21. M. Mikikian, L. Boufendi, A. Bouchoule, H.M. Thomas, G.E. Morfill, A.P. Nefedov, V.E. Fortov, The PKENefedov Team, New J. Phys. 5, 19 (2003)

22. C. Arnas, A.A. Mouberi, J. Appl. Phys. 105, 063301 (2009)

23. C. Szopa, G. Cernogora, L. Boufendi, J.J. Correia, P. Coll, Planet. Space Sci. 54, 394 (2006)

24. G.S. Selwyn, J. Singh, R.S. Bennett, J. Vac. Sci. Technol. A 7, 2758 (1989)

25. A. Bouchoule, L. Boufendi, J. Hermann, A. Plain, T. Hbid, G. Kroesen, W.W. Stoffels, Pure Appl. Chem. 68, 1121 (1996)

26. J. Winter, Plasma Phys. Control. Fus. 46, B583 (2004) 
M. Mikikian et al.: Dusty plasmas: synthesis, structure and dynamics of a dust cloud in a plasma

27. S.I. Krasheninnikov et al., Plasma Phys. Control. Fus. 50, 124054 (2008)

28. C. Arnas, A. Mouberi, K. Hassouni, A. Michau, G. Lombardi, X. Bonnin, F. Bénédic, B. Pégourié, J. Nucl. Mater. 390-391, 140 (2009)

29. M. Cavarroc, M. Mikikian, G. Perrier, L. Boufendi, Appl. Phys. Lett. 89, 013107 (2006)

30. P. Roca i Cabarrocas, P. Gay, A. Hadjadj, J. Vac. Sci. Technol. A 14, 655 (1996)

31. A. Dutta, S.P. Lee, Y. Hayafune, S. Hatatani, S. Oda, Jpn J. Appl. Phys. 39, 264 (2000)

32. J. Robertson, Mater. Sci. Eng. R 37, 129 (2002)

33. A.P. Nefedov et al., New J. Phys. 5, 33 (2003)

34. L. Boufendi, J. Gaudin, S. Huet, G. Viera, M. Dudemaine, Appl. Phys. Lett. 79, 4301 (2001)

35. A. Bouchoule, L. Boufendi, Plasma Sources Sci. Technol. 3, 292 (1994)

36. M. Cavarroc, M.C. Jouanny, K. Radouane, M. Mikikian, L. Boufendi, J. Appl. Phys. 99, 064301 (2006)

37. M. Cavarroc, M. Mikikian, Y. Tessier, L. Boufendi, Phys. Plasmas 15, 103704 (2008)

38. M. Mikikian, M. Cavarroc, L. Couëdel, L. Boufendi, Phys. Plasmas 13, 092103 (2006)

39. M. Mikikian, L. Couëdel, M. Cavarroc, Y. Tessier, L. Boufendi, IEEE Trans. Plasma Sci. 36, 1012 (2008)

40. W.L. Nighan, W.J. Wiegand, Phys. Rev. A 10, 922 (1974)

41. A. Descoeudres, L. Sansonnens, C. Hollenstein, Plasma Sources Sci. Technol. 12, 152 (2003)

42. Y. Kurimoto, N. Matsuda, G. Uchida, S. Iizuka, M. Suemitsu, N. Sato, Thin Solid Films 457, 285 (2004)
43. S. Iizuka, K. Sakuta, W. Suzukawa, K. Kato, T. Gohda, Phys. Plasmas 13, 103502 (2006)

44. Y.F. Li, U. Konopka, K. Jiang, T. Shimizu, H. Höfner, H.M. Thomas, G.E. Morfill, Appl. Phys. Lett. 94, 081502 (2009)

45. G.E. Morfill, H.M. Thomas, U. Konopka, H. Rothermel, M. Zuzic, A. Ivlev, J. Goree, Phys. Rev. Lett. 83, 1598 (1999)

46. J. Goree, G.E. Morfill, V.N. Tsytovich, S.V. Vladimirov, Phys. Rev. E 59, 7055 (1999)

47. M. Mikikian, L. Couëdel, M. Cavarroc, Y. Tessier, L. Boufendi, New J. Phys. 9, 268 (2007)

48. V. Land, W.J. Goedheer, New J. Phys. 9, 246 (2007)

49. I. Stefanović, C. Scharwitz, E. Kovačević, J. Berndt, J. Winter, IEEE Trans. Plasma Sci. 36, 1018 (2008)

50. M. Mikikian, M. Cavarroc, L. Couëdel, Y. Tessier, L. Boufendi, Phys. Rev. Lett. 100, 225005 (2008)

51. A.V. Ivlev et al., Phys. Rev. Lett. 90, 055003 (2003)

52. L. Couëdel, M. Mikikian, L. Boufendi, A.A. Samarian, Phys. Rev. E 74, 026403 (2006)

53. L. Couëdel, A.A. Samarian, M. Mikikian, L. Boufendi, Phys. Plasmas 15, 063705 (2008)

54. L. Couëdel, A.A. Samarian, M. Mikikian, L. Boufendi, EPL 84, 35002 (2008)

55. L. Couëdel, A. Mezeghrane, A.A. Samarian, M. Mikikian, Y. Tessier, M. Cavarroc, L. Boufendi, Contrib. Plasma Phys. 49, 235 (2009)

56. L. Couëdel, A. Mezeghrane, M. Mikikian, Y. Tessier, A.A. Samarian, C. Cuthbert, L. Boufendi, J. Electrostat. 67, 625 (2009) 